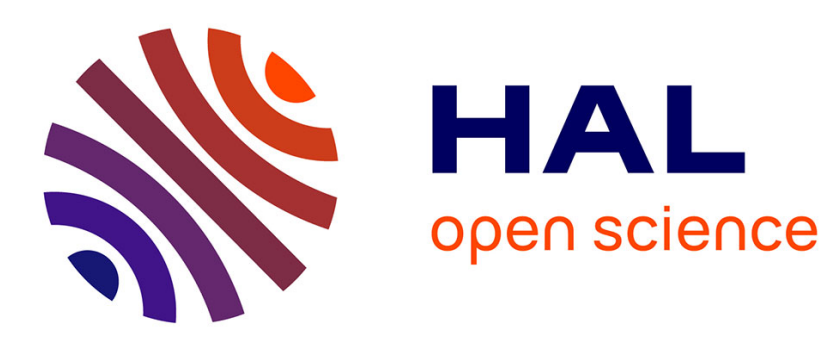

\title{
Une enquête sur les enceintes gauloises de l'Ouest et du Nord
}

\author{
Paul-Marie Duval
}

\section{To cite this version:}

Paul-Marie Duval. Une enquête sur les enceintes gauloises de l'Ouest et du Nord. Gallia - Fouilles et monuments archéologiques en France métropolitaine, 1959, 17 (1), pp.37-62. 10.3406/galia.1959.2255 . hal-01924481

\section{HAL Id: hal-01924481 \\ https://hal.science/hal-01924481}

Submitted on 3 Mar 2020

HAL is a multi-disciplinary open access archive for the deposit and dissemination of scientific research documents, whether they are published or not. The documents may come from teaching and research institutions in France or abroad, or from public or private research centers.
L'archive ouverte pluridisciplinaire HAL, est destinée au dépôt et à la diffusion de documents scientifiques de niveau recherche, publiés ou non, émanant des établissements d'enseignement et de recherche français ou étrangers, des laboratoires publics ou privés.

\section{(이) $\$$}

Distributed under a Creative Commons Attribution - NonCommercial - NoDerivatives| 4.0 


\title{
UNE ENQUETTE SUR LES ENCEINTES GAULOISES DE L'OUEST ET DU NORD
}

\author{
par M. Paul-Marie Duval
}

Les fortifications gauloises sont si nombreuses qu'on n'a pu, jusqu'à présent, leur consacrer une étude synthétique fondée sur un dénombrement complct, sur une analyse détailléc. Cé qu'Adrien Blanchet a réussi en 1907 pour les enceintes urbaines de la Gaule romaine n'a pas été tenté pour celles, beaucoup plus nombreuses et généralement moins distinctes, de la Gaule indépendante. La prospection, pourtant, a été active et fructueuse depuis le milieu du XIX ${ }^{\mathbf{e}}$ siècle. Au début, on tenait la plupart de ces ouvrages pour des "camps de César", par une erreur de perspective dont l'archéologie officielle du Second Empire fut en partie responsable. Il a fallu les trois premiers volumes de l'Histoire de la Gaule de Jullian, puis les chapitres du Manuel d'archéologie de Déchelette et Grenier (tomes IV et V), pour restituer à leurs auteurs gaulois le majorité de ces enceintes. Cependant l'enquête proprement archéologique se poursuivait lenlement dans toute la France. Des fouillcs s'effectuaient sur des sites privilégiés par l'éclat de leur destinée ou la consistance de leurs vestiges : Alésia, Gergovie, Bibracte ; un type de rempart proprement celtique, dit murus Gallicus, se définissait; les monographics locales, les répertoires départementaux se multipliaient ; le Dictionnaire archéologique de la Gaule décantait et condensait les résultats dans ses fascicules successifs (1875-1923). La difficulté de dater les enceintes préromaines avait provoqué la décision officielle d'inventorier tout ce qui ressemblait à une fortification de terre et de pierre sèche paraissant tant soit peu antique : ce fut l'œuvre de la "Commission pour l'étude des enceintes préhistoriques et fortifications anhistoriques ", qui publia ses listes d'inventaires par départements dans le Bullelin de la Société préhistorique française à partir de 1907 (jusqu'en 1920), avec d'utiles bibliographies. Ces matériaux étaient là rassemblés pour des enquêtes plus poussées, voire pour des synthèses qui renouvelleraient, en les précisant pour une région donnée, les exposés généraux de Caumont, Déchelette, La Noë, Alexandre de Mortillet, Castagné et Alexandre Bertrand.

Voici la première de ces synthèses, qui est due, curieusement, à nos confrères britanniques, naturellement intéressés par la partie du continent qui regarde 
leur île. Sir Mortimer Wheeler a depuis longtemps fait porter ses recherches sur les fortifications antiques de l'Occident : on lui doit un mémoire qui a fait datc, sur les cnceintes gallo-romaines d'Arles, dont il a distingué les remaniements successifs ${ }^{1}$. Il s'attaqua on 1935 aux enceintes préromaines de la GrandeBretagne méridionale et, après avoir exploré celle de Maiden Castle, d'un type particulier fait de remparts multiples protégeant une position dominante, il formula l'hypothèsc que cc système de défense, représenté dans le Dorsetshire et le Cornwall, devait avoir son origine sur le continent gaulois ${ }^{2}$ : les rapports étroits des peuples maritimes de la Gaulc avec ceux de la Bretagne à l'époque protohistorique, les liens resserrés par la résistance commune à l'invasion romaine, l'exil possible des Vénètes en Cornouailles après la catastrophe de 56 , suggéraient en effet l'hypothèse et fournissaient une vue historique à l'enquête archéologique. Sir Mortimer, poursuivant un objectif précis, mit sur pied une mission d'études avec l'accord du gouvernement français et, après une préparation attentive, conduisit en 1938 et 1939, au cours de l'été, deux campagnes de recherches et de sondages patronnées par la Société des Antiquaires de Londres et l'Université de Londres; quelques collaborateurs français y participèrent. Les départements côtiers et limitrophes furent visités, de l'embouchure de la Loire à celle de la Somme et l'enquête se développa plus largement vers l'intérieur : sur 200 sites retenus pour examen, 93 furent jugés dignes d'une reconnaissance détaillée, les collections des musées furent également étudiées, et les résultats dépassèrent l'espérance. Venue chercher sur le continent les prototypes éventuels des fortifications insulaires, la mission britannique accumula une foule d'observations qui précisaient souvent de façon nouvelle ce qu'on savait des fortifications gauloises de l'intérieur. La guerre interrompit l'enquête mais un rapport préliminaire, publié en français et en anglais ${ }^{3}$, exposa les résultats principaux, dont l'intérêt faisait souhaiter la préparation ultérieure d'un rapport détaillé.

Celui-ci paraît aujourd'hui, en langue anglaise, sous les auspices de la Société des Antiquaires de Londres, après des vérifications rendues nécessaires par les bouleversements de terrains dus à la gucre et par le temps écoulé ; elles furent faites en 1954, 1955 et 1956, notamment en Armorique, avec lc concours de nos Directeurs des Antiquités. L'ouvrage est essentiellement un

(1) R. E. M. WheELER, The Roman Townwalls of Arles, Journal of Roman Sludies, XVI (1926), p. 174-194, pl. XXI-XXIII.

(2) R. E. M. Wheeler, Maiden Castle, Dorsel, Reports of the Research Committee of the Society of Anliquaries of London, XII, Oxford, 1943.

(3) R. E. M. Wirecier, Les camps de l'Age du Fer dans le Nord-Ouest de la France et le SudOuest de la Grande-Bretagne, Rev. arch., $6^{\mathrm{e}}$ série, XIII (1939), p. 103-124, fig. et pl. (= Iron $\Lambda$ ge Camps in Northwestern France and Southwestern Britain, Anliquily, XIII (1939), p. 58-79, fig. 1-8, pl. I-VIII). 
inventaire analytique départemental et un compte rendu de fouilles mais une partic synthétique dégage d'abord les caractères des enceintes et institue des catégories. C'est un ouvrage de référence, dont le titre Hill-Forts of Northern France "Forts de collines de la France du Nord " exprime le caractère principal de ces enceintes gauloises de terre et de picrre sèche, plantées presque toujours en position dominante, - falaise, promontoire, colline ou plateau, que ce soit sur le littoral ou dans l'intérieur, en Bretagne ou en Normandie, en Picardie ou en Artois4. La contribution est importante à l'histoirc des peuples gaulois de ces régions : oppidums de l'Indépendance, rapports avec la Bretagne, puissante organisation défensive de la guerre des Gaules, sort de ces défenses à l'époque romaine. Chaque enceinte fait l'objet d'une notice qui précise souvent nos connaissances à son sujet. Cinq sites ont été explorés de façon à fournir des points de repère typologiques et chronologiques, des identifications nouvelles sont proposées pour certains centres importants et plusieurs sites nouveaux sont repérés, qui avaient échappé aux inventaires. Deux types originaux de fortifications sont définis, le type " vénète » à défenses multiples, le type "belge " à grand fossé plat; la question du murus Gallicus fait l'objet d'une mise au point en appendice. Quatre cartes de répartition groupenl les enceintes de même nature, une bibliographie abondante est présentée de façon analytique ${ }^{5}$; enfin des indications précicuses se dégagent de tout cela pour des fouilles futures.

Tel qu'il se présente, l'ouvrage est, une somme de documents, bien présentée et bien illustrée, précédée de pages suggestives qui mériteraient d'être traduites dans une revue française. On sait que la "field archaeology" a acquis en GrandeBretagne ses titres de noblesse : adaptée à la recherche (toujours délicate, et souvent ingrate) d'ouvrages étrangers à l'architecture proprement dite et le plus souvent invisibles à la surface du sol où d'insensibles mouvements de terrain ne les signalent pas à première vue, clle y a été fortement secondée par la photographie aérienne. L'histoire de la Gaule vient de bénéficier, autant que celle de

(4) Sir Mortimer WheELer et Katherine M. Richardson, Hill-Forls of Northern France, Appendice sur les Muri Gallici par M. Aylwin Coтton, Reports of the Research Committee of the Sociely of Anliquaries of London, XIX, Oxford 1957, $230 \mathrm{p}$. in-4 ${ }^{\circ}, \mathrm{L}$ pl. dont 2 cartes, 35 fig. donl 2 carles. Index. - On trouvera des comptes rendus de cet ouvrage notamment dans les Eludes (ieltiques, VIII, 2 (R. Jorrroy), la Revue $d u$ Nord, XL, 160, 1958 (E. WiLL), les Annales de Brelagne, les Annales de Normundie.

(5) P. 134-158, bibliographie analytique, et p. 217-225, bibliographie concernant les muri Gallici. A la première auraient pu figurer le Giuide illustré des campagnes de César en Gaule de L.-A. Constans (1926), les tomes II et III (1908-1909) de l'Histoire de la Gaule de Jullian, le Diclionnaire archéologique de la Gaule (1875-1923), les propres travaux de Sir Mortimer Whecler sur la question. $\Lambda$ la seconde, le Dictionnaire archéologique de la Gaule est donné comme ayant paru de 1875 à 1878 seulement, alors que le dernier fascicule est de 1923 ; et le Commandant Émile Espérandieu est appelè " le Comte Émile... ». - La Revue archéologique citée p. 12, n. 2, p. 130, n. 1, est en réalité lá Revue archéologique de l'Est el $d u$ Centre-Est. La superficie d'un certain nombre de sites fortifiés n'est pas indiquée ( $\mathrm{n}^{\circ \mathrm{s}} 3,13,23,33,59$, $63,69,72,92,126)$. 
la Bretagne, de cette expérience acquise outre-Manche et nous apprécions à sa juste valeur, qui est grande, cette contribution à l'histoire d'une antique alliance : il convient de la présenter ici en quelque détail à nos archéologues, qui auront à se servir souvent d'un ouvrage désormais indispensable à lcurs recherches. Nous classcrons sommairement d'après les cartes qui l'illustrent et les indications synthétiques qui le précèdent les résultats de l'inventairc qui nous est offert (voir la carte générale à la fin, fig. 2) ${ }^{6}$.

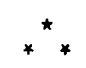

Deux critères principaux sont à la base du classement: Ia superficie, qui traduit l'importance de l'oppidum et généralement celle de son peuple; le système de fortification ou, plus largement, de défense, y compris les avantages du terrain (pour ce deuxième critère la technique du murus Gallicus n'est qu'une distinction supplémentaire car elle concerne seulement le rempart et non la forme de l'oppidum ou l'ensemble des défenses). L'appartenance à unc région déterminée ou à un ensemble de peuples de même caractère d'une part, la date d'autre part permettent d'établir des subdivisions à l'intéricur des catégories principales. Nous choisissons ici la superficie comme critère essentiel et, plutôt que le classement départemental qui est celui de l'inventaire? tionnel des cités gauloises, malgré l'incertitude qui règne souvent sur leurs limites. Nous considérons comme de grands oppidums de peuplade, d'accord avec les Auteurs de l'enquête, ceux dont la superficie est supérieure à une dizaine d'hectares ; si la plupart d'entre eux les dépassent de beaucoup (le plus

(6) Voici le plan de l'ouvrage, dont les Auteurs qualifient la composition d'u illogique mais, on l'espère commode " (Préface) - et cet espoir n'est nullement déçu - : Introduction: historique de la mission et de ses différents travaux. - Première partie: synthèses. I. Ies lrois calégories d'enceintes en lerre dans la France du Nord: a) Oppida de tribus de la série "le Petit Celland", b) Château-forts de falaises de la série "Castel-Coz ", c) Remparts belges de la série "Fécamp "; - II. Jules César el l'archéologie dans le Nord de la Gaule. -- Deuxième parlie: malériaux. III. Cinq sites fouillés en 1938-19.39: 1) Le Camp d'Artus, Huelgoat, 2) Le Chatellier, Le Petit Celland, 3) Kercaradec, Penhars, 4) Le Camp du Canada, Fccamp, 5) Le Catelier, Duclair ; - IV. Carrel de croquis de cérumique; - V. Caiulogue ("gazelleer ") des hauls-lieux forlifiés et structures étroilemenl apparenlées en Bretagne, Normandie et régions adjacentes. - VI. Bibliographie. - Appendice : Muri Gallici, par M. Aylwin Cotron, avec bibliographie. Index (un peu sommaire).

Les quatre cartes qui expriment le classement des enceintes sont : pl. XLIII (p. 103), les 93 enceintes répertorices (ici, fig. 2 ); pl. I (p. 1), seulement les grands oppidums, avec sigle particulier pour la série belge; fig. 1 (p. 5), seulement les châteaux-forts de falaise de la côte vénète ; fig. 35 (p. 165), les enceintes du type murus Gallicus, en France, Belgique, Suisse et Allemagne. - De belles photographies montrent les sites et les fouilles (notamment celles de muri Gallici avec les traces de poutres).

(7) La prospection a porté sur les départements (indiqués par des chiffres romains sur la carte de la pl. XLIII-V - ici la légende de la fig. 2) qui couvrent en gros la Bretagne, la Normandie, la Picardie et une partie de l'Artois. 
grand de tous, ancêtre de Lisieux, n'a pas moins de 162 hectares), un grand nombre sont, en revanche, très loin de les atteindre ${ }^{8}$.

1. Grands oppidums : Chef-Lieu, Quartier génḱral ou camp retranché, PLACE FORTE SECONDAIRE D'UNE CITÉ

Une série d'enceintes se détachent de la masse, par lcurs fortes dimensions et, secondairement, par leur système de fortification et leur répartition géographique. Quelques-uns de ces oppidums sont d'une importance et occupent une situation qui les désignent comme des chefs-licux de peuplade; d'autres ont la fortification hâtive, voire inachevée, d'un refuge temporaire, d'un quartier général ou d'un camp retranché principal ${ }^{8}$ bis ; mais d'autres enfin apparaissent comme les oppidums secondaires d'une peuplade, qui peut en posséder un certain nombre. La plupart occupent des situations dominantes.

Deux groupes se distinguent ${ }^{9}: 1^{0}$ celui des oppidums centraux d'une peuplade, destinés à abriter ses forces vilales en des circonstances criliques, soit que l'enceinte ait déjà existé, protégeant par exemple un chef-lieu, soit qu'elle ait été construite pour ces circonstances exceptionnelles : les indices chronologiques désignent, pour ce second cas, la réaction de 56 à l'occupation romaine, à l'Ouest de la Seine. Il sont du type traditionnel, avec fossé en V, parfois le murus Gallicus, et il n'y a pas de grands oppidums d'un autre type à l'Ouest de la Seine. A l'Est, chez les Belges, on les retrouve seulement à l'Est de Beauvais (sauf une exception) et au Nord de la Somme. Là, occupant parfois en plaine la boucle d'une rivière, ils se mêlent à l'autre type, proprement belge, de fortification de hauteur. - $2^{\circ}$ Ce type règne, lui, dans tout le pays belge, entre Seine et Somme. Il est caractérisé par un large fossé, plat ct, très différent de l'oppidum à murus Gallicus, se trouve en plusicurs exemplaires sur le territoire d'une même peuplade : il parait représenter la réaction proprement

(8) Nous exprimons en hcctares et ares les superficies données en acres anglais. L'acre équivaut à 40,5 ares, soit 4050 mètres carrés (tandis que l'ancien acre français valait 52 ares) : afin que nos chiffres ne soient pas d'une précision souvent fallacieuse, traduisant des estimations données dans la plupart des cas à l'acre ou au demi-acre près, donc à 20 ares près au maximum, nous les arrondirons à l'hectare ou au demi-hectare le plus proche, donc à moins de 25 ares près. Par excmple, pour 30 acres $=12$ hectares 15 ares : 12 ha ; pour 40 acres $=19$ hectares 44 ares : 19 ha, 50. En revanche pour les superficies inférieures à 3 hectares, nous donnerons le plus souvent l'équivalence exacte au chiffre rond anglais ainsi que pour un chiffre rond, quelle que soit son importance (50 acres: $20 \mathrm{ha}, 25$ ).

$(8$ bis) Cette explication par le rassemblement au centre de la tribu a été développée par Sir Mortimer Wheeler à propos des enceintes du Yorkshire, The Stanwick Forlifications, Reporls of... the Sociely of Anliquaries of London, XVII, Oxford, 1954.

(9) Carte, pl. I. 
belge aux invasions de 57/56 et même de 51 , la parade technique de ces régions aux machines de siège de la légion. Quelques exemples se trouvent à l'Est de Beauvais.

1. Grands oppidums centraux de peuplades ou camps retranchés

a) A l'Ouest de la Seine : type traditionnel à fossé en V.

Ils occupent une hautcur, plateau, promontoire, ou falaise surplombant une vallée.

\section{Abrincalui ${ }^{11}$ :}

*(43) Le Petit-Celland : Camp du Châtellier (Manche) ${ }^{11}$ : à 8 kilomètres à l'Est d'Avranches, sur la Sée ; inventaire B.S.P.F. XIII (1916), liste LII, 162; D.A.G., Petit-Celland (Le). Fouillé en 1938-39, sert de prototype au groupe $1^{12}$. Connu depuis 1840 au moins, longtemps idenlifié avec le camp de Sabinus pendant la campagne de 56 contre Viridorix. Murus Gallicus avec pieux verticaux dans la façade. 19 ha, 50. Monnaies gauloises. Inachevé : camp principal de Viridorix en 56 , brûlé par Sabinus en cette même année?

\section{Osismi:}

*(31) Huelgoat : Camp d'Artus (Finistère) : sur l'Aune ; inv. B.S.P. F.XI (1914), liste XXXI, 157 ; D.A.G., Huelgoat. Fouillé en 1938-39, comporte une division intérieure isolant environ 4 hectares $^{13}$. Murus Gallicus. 30 ha, 50. Grand, occupé peu longtemps, a dû être le quartier général des 0 sismi en 56 , la fortification réduite pouvant dater de 51 .

\section{Coriosolitae ou Veneti :}

(8) Guégon : Camp de Lescouais ou Lescouct (Morbihan) : à 7 kilomètres à l'Ouest de Josselin ; inv. B.S.P.F. XIV (1917), liste XVIII, 456. Au moins 24 ha. 50, peut-être plus de 32 ha, 50. A dû être le quartier général des Curiosolilae en 56, plutôt que celui des Veneti, qui, ayant leur puissance sur mer et leurs forteresses sur le littoral, n'avaient sans doute pas d'oppidum central dans l'intérieur.

Redones:

(42) Landéan : oppidum du Poulailler (Ille-et-Vilaine) : à la lisière ouest de la Forêt de Fougères ${ }^{14}$; P. Banéat, Le département d'Ille-et-Vilaine, II (1927). Rempart à parement de pierre, peut-être murus Gallicus. 20 ha, 25. Grandement inachevé, a dû être construit trop tard pour être le quartier général des Redones en 56.

\section{Coriosolitae :}

(40) Saint-Coulomb : la Ville des Mues (Ille-et-Vilaine) : sur la Pointe du Meinga, à 3 kilomètres au N.-O. de Saint-Coulomb et à 12 kilomètres au N.-E. de Saint-Malo ;

(10) Sans doute clients des Unelli du Cotentin. Peut-être les Ambibarii de César ?

(11) Le numéro de tête entre parenthèses est celui de l'inventaire Wheeler-Richardson. Le premier nom est généralement celui de la commune. L'astérisque distingue les cinq sites explorés méthodiquement en 1938 et 1939.

(12) Compte-rendu des fouilles, p. 38-54, plan (pl. XVI), relevés et photographies, monnaies (pl. XVII-XXV), clous et poteries (fig. 7-10).

(13) Compte-rendu des fouilles, p. 23-38, plan (pl. II), relevés et photographies (fig. 3, 4, 4a, (pl. III-XV), poteries (fig. 5-6).

(14) I'lan, fig. 32, p. 114. Notice sur le murus Gallicus possible, p. 208. 
inv. B.S.P.F. XI (1914), liste XXXVII, 279. Visité en 1939 et 1954. Éperon barré par un rempart à revêtement de pierre avec traces de bois brúlé qui représente peut-être le poutrage d'un murus Gallicus ${ }^{15}$. De 14 ha, 50 à 16 ha. Nous ajoutons cette enceinte à la liste des grands oppidums à cause de la superficie et de la possibilité d'un murus Gallicus; la situation excentrique n'est pas celle d'un oppidum de peuplade mais il peut s'agir d'une place importante des Redones sur le front de mer.

Unelli :

(45) Lithaire : Le Grand Moncastre (Mont-Castre), Camp de César (Manche) : à l'Ouest de Garentan ; inv. B.S.P.F. XIII (1916), liste LII, 163. Sondages 1839, 1862. De 16 à 20 ha. Monnaies gauloises, poteries : a dû être l'oppidum central de la puissante peuplade des Unelli.

N. B. - Tourlaville : le Fossé-Catel (Manche) : camp à rechercher d'une étendue comparable à celle du précédent; cf. inv. B.S.P.F. XIII (1916), liste LII, $162^{16}$.

\section{Baiocasses:}

(50) Castillon (Galvados) : sur la Drôme, contient le village et le château de Castillon; inv. B.S.P.F. X (1913), liste $282 ;$ D.A.G., Gastillon. 44 ha 50. Trésor de monnaies gauloises. Devait être l'oppidum central des Baiocasses.

(49) Saint-Jean-de-Savigny : Le Grand Gâtel (Manche) : à 11 kilomètres au N.-E. de Saint-Lô ; inv. B.S.P.F. XIII (1915), liste LII, 162 (identifié avec "La Butte des Romains, Cérisy-la-Forêt »). Rempart à parement de pierre, peut-être murus Gallicus. 5 ha, 50 : nettement plus petit que tous ceux de la série, a dû être pourtant comme eux, par les avantages de sa position (promontoire) et de sa fortification, un oppidum important des Baiocasses ${ }^{17}$. Celui de Castillon était cependant beaucoup plus vaste.

Lexovii :

(56) Saint-Désir (Lisieux) : Camp du Castellier (Calvados) : à 3 kilomètres au S.-O. de Lisieux, décrit par Caumont en 1834 ; inv. B.S.P.F. X (1913), liste XVI, 283. Traces intermittentes d'une immense enceinte ${ }^{18}$, murus Gallicus, clous. Environ 162 ha : le plus grand de tous ces oppidums (probablement en partie un camp-refuge), certainement le chef-lieu des Lexovii, Noviomagus, qui devait ensuite occuper l'emplacement de Lisieux.

Ainsi, à l'Ouest de la Seine, les peuples gaulois "armoricains " paraissent s'être ralliés chacun en un point central de son territoire ; s'il n'était pas déjà leur chef-lieu et fortifié en tant que tel, il fut alors entouré de défenses qui empruntèrent souvent la technique du murus Gallicus, appliquée dans le reste de la Gaule, à l'exception de la Belgique et de la Narbonnaise. Cette mise en état

(15) Cf. la notice sur le murus Gallicus possible, p. 208, et la notice, p. 113.

(16) Cf. p. 116, Nole.

(17) Cf. p. $2-3$; ne figure pas, toutefois, en raison de sa faible superficie, sur la carte des grands oppidums, pl. I.

(18) Plan, fig. 33, p. 118, d'après une vue aérienne. Notice, p. 203-204, à propos du murus Gallicus, constaté et fouillé vers 1880 . 
de défense de l'Armorique "sur une échelle formidable " répond à la menace romaine de 57-56 mieux qu'à aucune autre conjoncture.

b) A l'Est de la Seine : coexistence du type traditionnel avec le type "belge". Deux genres de situation différents : dominante, comme précédemment; en plaine, à l'abri d'une rivière.

Situation dominante:

Veliocasses:

(68) Caudebec : Camp de Calidu ou Caledon (Seine-Maritime) : à l'Ouest de Caudebec, avec accès facile à la Seine; inv. B.S.P.F. XVI (1919), liste LXXVII, 186; D.A.G., Caudebec. Le fossé est plat mais petit et ne fait qu'un avec le rempart. De 20 à 40 ha. Monnaies gauloises el romaines, tuiles. Site très important ${ }^{19}$, que nous intégrons dans la série des grands oppidums ; c'est le seul en zone belge à l'Ouest de Beauvais, qui ne soit pas du type belge (v. plus loin).

\section{Bellovaci:}

(81) Bailleul-sur-Thérain : le Mont-César (Oise) : à 13 kilomètres à l'E.-S. E. de Beauvais, sur le Thérain; inv. B.S.P.F. XIV (1917), liste LXI, 469; D.A.G., Bailleulsur-Thérain. Fouilles 1878 d'un tumulus, monnaies et poteries gauloises et gallo-romaines, fibule de La Tène. 35 ha. A pu être le chef-lieu des Bellovaci, avant le transfert sur le site de Beauvais et même avant l'arrivée des Belges.

Suessiones:

(83) Vieux-Moulin : Camp Romain (Oise) : à Saint-Pierre-en-Chastre, à 6 kilomètres au S.-E. de Compiègne, en forêt; inv. B.S.P.F. XIV (1917), liste LXI, 469 ; D.A.G., Vieux-Moulin (Le); Grenier, Manuel, V, 1, p. 194-198, fig. 13. Fouilles sous Napoléon III, présentation en 1862 par Viollet-le-Duc à la façon des fortifications romaines. 26 ha, 50. Monnaies gauloises. N'est pas central dans le territoire des Suessiones mais a dû être un oppidum important ; v. la discussion dans Jullian, Hist. de la Gaule, III, p. 549, n. 1.

(84) Montigny-l'Engrain : Le Châtelet (Aisne) : au S.-S.O. de Vic-sur-Aisne, entre Courtieux et Cessons-le-Long; inv. B.S.P.F. X (1913), liste II, 101 ; D.A.G., Montigny-Lengrain. Fouilles 1887 (Vauvillé). Rempart à revêtement extérieur de pierre ; de longs clous suggèrent la présente d'un murus Gallicus ${ }^{20} .9$ ha. Monnaies gauloises. Peut-être un castellum plutôt qu'un oppidum, mais qui mérite, malgré sa faible superficie, d'être adjoint à la série des grandes enceintes à cause de son murus Gallicus.

Viromandui:

(91) Vermand (Aisne) : contient le village actuel, à 10 kilomètres à l'Ouest de Saint-Quentin ; inv. B.S.P.F. X (1913), liste II, 163. Il y a peut-être au S.-O. un fossé du type "Fécamp " (v. plus loin). 15 ha. Monnaies gauloises et gallo-romaines (Tibère).

(19) Cf. p. 122 ; ne figure pas, toutefois, sur la carte des grands oppidums, pl. I.

(20) Ia notice technique, p. 205-206 est affirmative sur ce point, plus que celle de la p. 129. 
Devait être le chef-lieu des Viromandui dont il perpétue le nom, avant le transfert sur le site de Saint-Quentin, où s'éleva Augusta Viromanduorum.

? Ambiani:

V. plus loin, II, 2, Mareuil-Gaubert, $\mathrm{n}^{0} 76$.

Atrebates :

(93) Etrun : Camp de César (Pas-de-Calais) : touchant le village à l'Ouest, à 7 kilomètres au N.-O. d'Arras ; inv. B.S.P.F. XIV (1917), liste LXIII, 476. Le fossé du rempart était peut-être peu profond comme dans le type "Fécamp ". 20 ha, 25. C'est le seul oppidum connu chez les Atrebates, peut-être leur chel-lieu : y a-t-il un rapport entre les deux noms? (Cf. Etrœungt, près de Vervins, sur la route de Bavay, à l'emplacement de l'antique Duronum de la Table de Peutinger).

Nervii:

(92) Avesnes : Camp de César, Le Câtelet (Nord) : à 1 kilomètre à l'Est d'Avesnelles, faubourg oriental d'Avesnes; inv. B.S.P.F. XIV (1917), liste LX, 468 (Avesnelles); D.A.G., Flaumont-Waudrechies. On a fait état, avec raison, semble-il d'un murus Gallicus ${ }^{21}$. Une carrière ayant détruit une bonne partie de l'enceinte, la superficie, certainement grande, ne peut plus être estimée (D.A.G.: 14 ha 18 ares 60 centiares). Monnaies gauloises. Le chef-lieu gallo-romain, qui ne parait pas avoir de passé gaulois malgré son nom de Bayacum, est à une trentaine de kilomètres au Nord, à Bavai (Nord); les Nerviens ne paraissent pas avoir de villes : peut-être un oppidum au Grand Camp (Orchies)?.

\section{Remi :}

(89) Saint-Thomas : Le Vieux-Laon, Camp de César, Camp des Romains (Aisne) : à 19 kilomètres au S.-E. de Laon; inv. B.S.P.F., X (1913), liste II, 102; D.A.G., SainlThomas. Enceinte fouillée en 1887-1888. 32 ha, 50. A l'Ouest de l'entrée nord, on a isolé par un rempart transversal une petite enceinte en pointe, du type " Fécamp " pour les dimensions mais non pour le fossé, étroit et en $\mathrm{V}$, probablement antérieur à ce type, qui est d'époque césarienne; 6 ha, avec puits et traces d'occupalion permanente. Oppidum secondaire des Remi, qui pourrait être identifié avec Bibrax (v. plus loin, $\mathrm{n}^{\mathrm{o}} 88$ ) et dont on reprendrait l'exploration avec fruit.

Ainsi, à l'Est de la Scine, à une majorité d'oppidums scmblables à ceux de la série précédente et parfois comme eux à murus Gallicus, se mêle une minorité d'enceintes qui présentent peut-être un type nouveau, que nous appellerons plus loin le type "belge ".

En plaine, dans la boucle d'une rivière:

Remi:

(88) Guignicourt : Le Vieux-Reims (Aisne) : au confluent de l'Aisne et de la Suippe, à $17 \mathrm{~km} .700$ au N.-N.O. de Reims; inv. B.S.P.F. X (1919), liste II, 99 (Condé-surSuippe). Détruit en partie par le canal de l'Aisne en $1842: 111$ ha, 50.. Poteries gauloises, bracelet de bronze massif. Cet immense oppidum, situé sur la rive gauche de l'Aisne, dans

(21) V. la notice p. 209. 
la région marnienne, riche par ses échanges autant que par son agriculture à l'époque de La Tène, doit-il être identifié avec le chef-lieu des Rèmes, étant donnée sa vaste étendue, ou avec Bibrax, où la confédération belge assiégera en 57 les Remi alliés de César, qui les y secourut? Un camp romain a été identifié à $3 \mathrm{~km}$. 500 à l'O.-S.O., à Mauchamp, par Napoléon III (cf. Grenier, Manuel, V, 1, p. 191-194, fig. 11-12). Le camp de Sabinus pouvait être à Berry-au-Bac, à 7 ou 8 kilomètres au Nord du Vieux-Reims : 8 milles d'après B. G. II, 6, 1. Sur Bibrax, cf. D.A.G., Bibrax, et Jullian, Hist. de la Gaule, III, p. 253, n. 2 (v. plus haut, $\mathrm{n}^{0} 89$ ).

\section{Catuvellauni :}

La Cheppe : Le Vieux-Châlons, Le Camp d'Attila (Marne) ${ }^{22}$ : à $13 \mathrm{~km} .700$ au N.-E. de Châlons-sur-Marne, dans une boucle de la Noblette; D.A.G., Cheppe (La). Se trouve en dehors de la carte, fig. 2. Fortifications énormes à fossé en V. 20 ha, 25. Certainement le chef-lieu des Catuvellauni. Une peuplade portant le même nom, fixée en Bretagne dans le Hertforshire, a fortifié son oppidum (Weathampstead) d'une façon qui rappelle celle du Vieux-Châlons. Le nom de l'oppidum gaulois nous est inconnu, comme celui du chef-lieu gallo-romain qui devait prendre le nom des Catuvellauni, Châlons.

Ces oppidums de plaine, plus aptes que ceux des hauteurs aux aises de la vie citadine, plus propices aux exigences d'un urbanisme naissant, s'inscrivent dans une série qui comprend La Tène en Suisse, Manching en Bavière (murus Gallicus), peut-être la station commerciale de Chalon-sur-Saône et même Avaricum (Bourges), dont le terrain est toutefois moins plat (murus Gallicus). C'est l'oppidum en route vers l'urbs.

Ainsi, à l'Est de la Seine, une partie du pays belge possède de grands oppidums de peuplade, principaux ou secondaires, du même type que ceux de la région à l'Ouest du fleuve. Ce sont, souvent, les chefs-lieux de ces peuplades et ils sont, en tant que tels, plus anciens que la mise en défense provoquée par l'attaque romaine. Il comportent rarement le murus Gallicus. Certains attestent peut-être, par un large fossé à fond plat ( $\left.\mathrm{n}^{\text {os }} 91,93,89\right)$, le typc de défense qui règne à cette époque, dans le pays belge d'entre Seine et Somme et qui sera examiné ci-après. Mais, par opposition à cette enceinte belgc, c'est le murus Gallicus qui caractérise les plus puissantes des grandes enceintes qui viennent d'être passées en revue, surtout à l'Ouest de la Seine. La mise au point due à Mrs M. Aylwin Cotton apporte sur cette technique d'intéressantes précisions et un précieux répertoire.

Lc terme de murus Gallicus doit être réservé aux remparts de terre et de moellons, parementés de pierres et armés de poutres assemblées avec de longs clous (de 14 à 55 centimètres). Ils doivent être strictement distingués des remparts hallstattiens à poutrages de bois et des remparts germaniques et

(22) Hors de la carte de l'enquête, ne fait pas partie de l'inventaire mais est décrit p. 13-14. 
brittons plus récents, qui, tous sans clous, pourraient tenir debout sans le poutrage, contrairemenl au murus Gallicus. "Ici, la forme du poutragc ... cst celle décrite par César : les poutres transversales et longitudinales sont posées en couches alternées et fixées avec des clous à leurs points d'intersection. Les poutres transversales peuvent parfois être "attachées" (tied in) aux murs de façade en pierre. L'emploi des diagonales est rare (ou douteux) et on ne rencontre pas de poutres verticales dans les exemples classiques. Quand il y a un mur de revêtement extérieur, les poutres transversales pénètrent toujours. Chaque lit de poutrage est isolé par de la terre et des pierres dressées des lits supérieur et inférieur. Il n'y a pas de mur de façade d'ensemble continu : ce sont les intervalles entre les extrémités extérieures des poutres transversales qui sont dressés en paroi, et les alignements de têtes de poutres sont un lrail particulier de la façade. Quand ces poutres pourrissent, on laisse les trous vides " (p. 175). Ces enceintes datent de la première moitié du ${ }^{\text {er }}$ siècle av. J.-C. ; elles représentent le type de défense gaulois normal au début de la guerre des Gaules. Toutefois, cette technique s'est répandue hors de Gaule, où l'on en connaît maintenant quelques exemples ${ }^{23}$.

\section{Grands oppidums à enceinte de type belge, entre Seine et Somme.}

Ils occupent une position de hauteur, dominant parfois une vallée ou le littoral, à raison de plusieurs pour une même peuplade. Ces enceintes se distin-

(23) Voici, d'après la carte de répartition (fig. 35, p. 165), les exemples certains et supposés (") en dehors de ceux précédemment cités (dans l'ordre : 43, 31, 42*, 40*, 49*, 56, 84*,92*) et en dehors de la Narbonnaise, qui n'en comporte aucun. Une notice substantielle sur chaque cas accompagne cette étude très précise et bien documentée qui constitue l'Appendice, p. 159-226.

Segusiavi: Le Crêt-Chatelard (Saint-Marcel-de-Félines, Loire) ; Essalois (Loire); le Châtelard de Chazi (Saint-Georges-de-Baroille, Loiret) ; Jœuvres (Saint-Maurice-sur-Loire, Loire) ;

Lemovices: Le Puy-du-Tour (Monceaux, Corrèze); Villejoubert (Saint-Léonard, Haute-Vienne); Cadurci: Murcens (Lot), l'Impernal (Luzech, Lot);

Aedui : Bibracle - Mont-Beuvray (Saône-et-Loire et Nièvre) ;

Mandubii: Alesia - Alise-Sainte-Reine (Côte-d'Or) ;

Lingones: Verlillum - Vertault (Châtillon-sur-Seine, Côte-d'Or) ; Mont-Lassois (Vix, Côte-d'Or) ; Bois-vert (Lavilleuve, Côte-d'Or) ;

Bituriges: Avaricum - Bourges (Cher);

Pictones: Camp de Cornouin (Montmorillon, Vienne);

Andes: La Ségourie (Cholet, Maine-et-Loire);

Leuci: Boviolles (Meuse);

Petrocorii: Coulounieix (Dordogne);

Namnetes: Vue (Loire-Atlantique);

Aduatuci: Hastédon (Namur, Belgique);

Treviri: Ring d'Otzenhausen (Hunsrück, Allemagne);

Raurici : Tarodunum - Zarten (Freiburg-en-Brisgau, Allemagne) ; Mont-Terrible (Porrentruy, Berne, Suisse);

Helvetii: Engelhalbinsel (Berne, Suisse);

Vindelici (hors de Gaule) : Manching (Ingolstadt, Bavière, Allemagne);

Peuple d'Écosse : Burghead (Morayshire, Ecosse). 
guent des précédentes par les caractères suivants, reconnus pour la première fois en 1939: 10 préférence pour le site de promontoirc, coupé par un rempart (c'est ce que nous appelons couramment "l'éperon barré »); $2^{\circ}$ rempart très haut, de 6 à 9 mètres; très massif ; précédé d'un fossé large, à fond plat ou légèrement incurvé, scmblable à un canal et bordé du côté de l'extérieur par un talus en pente assez accentuée parfois renforcé d'une petite contrescarpe (fig. 1); $3^{0}$ entrées puissantes souvent flanquées de retours du rempart principal; $4^{0}$ sous-sol souvent argileux, mêlé de silex, peut-être choisi pour les ressources du sol en eaux de surface; $5^{\circ}$ tendance à inclure dans la fortification un accès facile à la mer ou à une rivière importante. La définition de ces caractères est peut-être l'apport le plus neuf de la présente enquête : on ne les trouve qu'en territoire belge, dans la basse vallće et au Nord de la Seine, dans les vallées de la Somme et de l'Aisne, soit chez les Veliocasses, Aulerci Eburovices, Caleti, Ambiani et Suessiones ${ }^{24}$. I es objets trouvés, notamment à Pommiers, indiquent une occupation pendant la première moitié du Ier sièclc av. J.-C. : comme le murus Gallicus est rare dans la région considérée, il est probable que ce type d'enceinte soit la parade proprement belge aux machines de siège romaines et que la séric soit donc d'époque césarienne, 57-51, ainsi que l'extension partielle de la technique aux grands oppidums de type traditionnel, plus haut présumée (nos 91 (?), $93(?), 89$ (?)). Il est possible que le duplex murus de l'oppidum central où se rassemblèrent les Aduatuci en 57 (B. G. II, 29) évoque ce genre d'ouvrage.

Caleti

*('11) Fécamp (commune : Toussaint) : Camp du Canada, Camp de César (SeineMaritime) : sur le littoral du Pays-de-Caux el accessible du port de Fécamp, face au Sussex ; à $3 \mathrm{~km}$. 200 de la mer, entre les ruisseaux de Valmont et de Ganzeville ; appelé Camp du Canada depuis 1700 au moins ; fouilles 1890 et $1939^{25}$; inv. B.S.P.F. XVI (1919), liste LXXVII, 187 ; D.A.G., Fécamp. Au moins 20 ha, 25. Abondance de tranchets néolithiques; monnaies gauloises, peut-être installation annexe d'un temple de type celtique; poterie sigillée et belge d'époque augustéenne. L'occupation gauloise a duré peu de temps.

("33) Bracquemont : Cité de Limes, Camp de César (Seine-Maritime) : sur le littoral du Pays-de-Caux, sur la falaise au N.-E. de Dieppe, constamment rongée par la mer; accès à la mer. Repéré en 1731, fouillé en 1822-1827, 1871, 1874, 1891, 1898, 1926 ; inv. B.S.P.F. XVI (1919), liste LXXVII, $186 ;$ D.A.G., Braquemont. La superficie était supérieure à l'actuelle, qui atteint encore $\mathbf{4 8} \mathbf{~ h a , ~} \mathbf{5 0}^{26}$. Restes d'un rempart plus

(24) V. toujours la carte des grands oppidums, pl. 1.

(25) Compte-rendu des fouilles, p. 62-75, plan (pl. XXVIII), relevés et photographies (fig. 2, 13, pl. XXIX-XXXVII), poteries et silex (fig. 14-19). p. 10.

(26) Plan sommaire, fig. 34 , p. 124, d'après une vue aérienne; profil de la fortification, fig. 2 , 
ancien à l'intérieur ; un temple de type celtique, découvert en 1822 , s'est abîmé depuis dans la mer; monnaies gauloises et romaines, poteries gauloises et romaines, fibules de La Tène III. (Profil du rempart et du fossé, fig. 1).

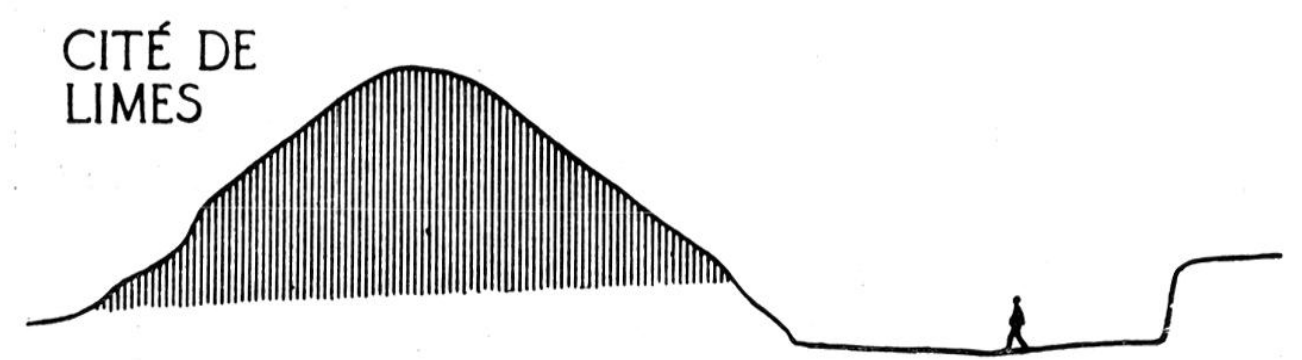

Fig. 1. - La Gité de Limes, Bracquemont, Seine-Maritime (fig. 2, no 73). Profil du rempart et de l'enceinte. (D'après Hill-Fort of Norlhern France, fig. 2, avec l'aimable autorisation de Sir Mortimer Wheeler).

(72) Veulettes : Le Câtelier, Le Tombeau de Gargantua (Seine-Maritime) : sur la falaise du Pays-de-Caux, à l'Ouest de la plage de Veulettes; inv. B.S.P.F. XVI (1919), liste LXXVII, 188 ; D.A.G., Veulettes. Superficie grandement diminuée par la mer. Monnaies romaines.

(74) Heugleville-sur-Scie (Seine-Maritime) : au cœur du pays calète, beaucoup plus petit et plus légèrement défendu que les oppidums maritimes. 3 ha. Malgré son exiguïté, doit être intégré à la série à cause du fossé.

(75) Incheville : Camp de Mortagne (Seine-Maritime) : sur le bord du plateau surplombant la Bresle, rive gauche, à 750 mètres de l'église d'Incheville; inv. B.S.P.F. XVI (1919), lisle LXXVII, $196 ;$ D.A.G., Incheville. Fouilles 1856 et de l'abbé Cochet : sépultures à inhumation et à incinération à l'intérieur et à l'extérieur de l'enceinte, certaines du Bas-Empire. 12 ha, l'un des plus petits de la série.

\section{Veliocasses:}

(61) Saint-Samson-de-la-Roque : Camp aux $\Lambda$ nglais (Eure) : dominant le Marais Vernier, ancien lit de la Scine ; inv. B.S.P.F. XI (1914), liste XXIX, 154. Le fossé plat à contrescarpe permet de l'intégrer à la série du type "Fécamp ». 97 ha : c'est de loin le plus vaste de la séric et cette ampleur l'apparente aux plus grands oppidums du type traditionnel.

(65) Sandouville : Camp de César (Seine-Maritime) : au confluent de la Seine et de l'Oudalle; inv. B.S.P.F. XVI (1919), liste LXXVII, 186;D. A. G., Sandouville. Rempart long d'environ 1 kilomètre. 81 ha. Monnaic gauloise.

(67) Caudebec (Seine-Maritime) : dominant la Seine, immédialement à l'Est de Caudebec. La forme plate du fossé permet de l'intégrer à la série malgré la faible superficie, d'ailleurs difficile à estimer : de 4 a 6 ha.

*('70) Saint-Pierre-de-Varengeville : Le Câtelier ou Châtelier, Les Portes de la Ville (Seine-Maritime) : à l'ancien confluent de la Seine et de la Sainte Austreberthe (aujourd'hui à sec), au Sud du village de Duclair, sur un sol d'argile à silex; inv. B.S.P.F. XVI (1919), liste LXXVII, 188; D.A.G., Saint-Pierre-de-Varengeville. On donne parfois, sans raison valable, le nom de Duclair, chef-lieu de canton, à ce sîte. Reconnu comme 
"camp gaulois " par l'abbé Cochet; fouilles 1939, interrompues par la déclaration de guerre ${ }^{27}$. Au moins deux périodes de construction : traces d'une première fortification à l'Est, hors de la fortification principale, enfin peut-être la pointe ouest. Revêtement de pierre au sommet du rempart, comme à Oldbury (Kent) et Poundbury (Dorsetshire) ${ }^{27 b i s}$. 10 ha. Occupé peu de temps : poteries et fibules de La Tène III, rien de gallo-romain. Les silex, très grossiers, peuvent appartenir à l'Age du Fer.

(63) Vernon : Camp de César, Camp romain, Camp de Mortagne (Eure) : à Vernonnet, dominant la rive droite de la Seine, sur un sol d'argile à silex ; inv. B.S.P.F. XI (1914), liste XXIX, 155. 64 ha, 50. Tessons de l'Age du Fer.

Aulerci Eburovices :

(62) Saint-Pierre-d'Aulils : Le Goulet, Le Pied d'Anglais, Le Trou aux Anglais (Eure) : rive gauche de la Seine; inv. B.S.P.F. XI (1914), liste XXIX, 154. Une porte secondaire mène droil au fleuve. 10 ha, l'un des plus petits de la série. Poterics galloromaines.

Ambiani:

(gry) Liercourt : Le Gatelier, Camp de César (Somme) : à 1600 mètres au N.-0. de Liercourt, surplombant la vallée de la Somme; inv. B.S.P.F. XVII (1920), liste LXXIX, 56. Le rempart S.-0., l'un des plus beaux de son espèce, est un exemple typique de la série belge, avec trace d'un fossé secondaire ${ }^{28} .32$ ha, 50. Fouilles du xix ${ }^{\mathrm{e}} \mathrm{s}$. : poteries "gauloise ", mérovingienne, médiévale ; traces d'occupation "néolithique ", "gauloise ", et "gallo-romaine"; monnaies des Antonins.

(79) La Chaussée-Tirancourt: Le Grand Fort, Camp de César (Somme) : à $10 \mathrm{~km} .500$ au N.-O. d'Amiens, dominant la vallée de la Somme ; inv. B.S.P.F. XVII (1920), liste LXXIX, 56 ; D.A.G., Chaussée Tirancourt (La). Coupe avant 1890 dans le rempart ${ }^{29}$. 20 ha, 25. Poterie "gauloise".

N. B. - (78) L'Étoile : Le Castelet, Camp de César (Somme) : près de l'église du village, entre les deux camps précédents sur la Somme moyenne; inv. B.S.P.F. XVII (1920), liste LXXIX, 56. Sondages du XIX ${ }^{\mathrm{e}} \mathrm{s}$. Rempart continu avec deux fossés à profil arrondi, poteries "gauloises ", gallo-romaines et mérovingiennes. 9 ha, 50. Vraisemblablement du type belge ; à préciser par des fouilles. Cf. Grenier, Manuel, V, 1, p. 193, n. 4.

\section{Suessiones:}

(86) Pommiers (Aisne) : immédiatement au N.-E. du village, à 5 kilomètres au N.-O. de Soissons, dominant la vallée de l'Aisne ; inv. B.S.P.F., X (1913), liste II, 101 ; D. A. G., Pommiers. Fouilles 1860, 1887 et suivantes, 1903-1904 (Vauvillé). $40 \mathrm{ha}, 50$. D'après les nombreuses monnaies gauloises et les monnaies romaines retrouvées, le site a été occupé de 70 environ à 51 , surtoul pendant la guerre des Gaules ; sous

(27) Compte rendu des fouilles, p. 75-83, plan (fig. 20 et pl. XXXVIII), relevés et photographies (fig. 2, pl. XXXIX-XLII), poteries, objets métalliques, silex (fig. 21-23).

(27 bis) Sur Oldbury, cf. J. B. Ward Perkins, Excavations on the Iron Age Hill-fort of Oldbury, near Ightham, Kent, Archaeologia XC, 1944, p. 128-176.

(28) Profil de la fortification, fig. 2, p. 10.

(29) Profil actuel, fig. 2, p. 10. 
Auguste, et moins régulièrement de Tibère au milieu du III $^{\mathrm{e}}$ siècle $^{30}$. Immédiatement au Nord, fortification à fossé en $\mathrm{V}$ qui paraît être également gauloise. Il y a tout lieu d'identifier ce grand oppidum à enceinte de type belge avec Noviodunum des Suessiones, point de ralliement de ce peuple en 57, que César ne put emporter propter latitudinem fossae murique allitudinem (B.G. $\quad$ ); v. Jullian, Hist. Gaule, III, p. 257, n. 3.

(85) Ambleny : Le Châtel (Aisne) : à $9 \mathrm{~km} .650$ à l'Ouest de Soissons, au N.-E. d'Ambleny; inv. B.S.P.F. X (1913), liste II, 98.8 ha, l'un des plus petits de la série : un castellum plutôt qu'un oppidum.

(87) Muret-et-Grouttes : Camp de César (Aisne) : à $14 \mathrm{~km}$. 500 au S.-S.E. de Soissons, englobant l'église et les ruines du château; inv. B.S.P.F. X (1913), liste II, 101. 16 ha.

Ces grands oppidums, spécifiquement belges, d'entre Seine et Somme, sont plus nombreux au sein d'une même peuplade que ccux du type traditionnel: au nombre de seize en tout, ils ne peuvent avoir été, comme les précédents, des centres de tribus. Ils ont été occupés surtout pendant la guerre des Gaules. Tenu compte aussi des fortifications partielles de même technique ajoutées aux autres grands oppidums, on voit se lever, aussi bien sur le rivage de la Manche que dans l'intéricur el particulièrement sur le bord des fleuves, la formidable résistance de la Gaule belgique qui se hérisse tout d'un coup de puissants bastions pour faire front aux légions romaines.

\section{Enceintes SEcondaires, DE faible SUPERficif:}

Elles se distinguent surlout du premicr groupe par lcur superficic, inférieure sans exception à 10 hectares, souvent même très faible, et par l'absence de système puissant de fortification, murus Gallicus ou rempart belge à fossé plat. Le groupe est remarquable par la densité et par une série particulière de châteauxforts de falaise, d'un type propre à la côte vénètc.

\section{Les châteaux-forts de falaises à défenses multiples des Veneti et des Osismi}

Les Vénètes étaient un peuple de marins, qui vivaient de la mer et entretenaient des relations avec l'île de Bretagne. Ils surent grouper en 56 sous leur commandement les peuples riverains de l'Océan et de la Manche et eurent même des alliés britanniques. César décrit leur mode de vic et de défense : groupés à l'abri de remparts sur les promontoires maritimes, ils se réfugiaient avec leur fortune sur leurs navires et communiquaient ainsi d'une forteresse à l'autre (B.G., III, 12 et 9$)$. Ces enceintes sont petites et certaines d'entre elles sont caractérisées par les remparts multiples barrant à sa base la langue de terre,

(30) Cf. Dr. J.-B. Colbert de Beaulieu, Peut-on dater par la numismatique l'occupation gauloise d'un oppidum? L'exemple de Pommiers (Aisne), Rev. arch. de l'Est et du Centre-Est, VI (1955), p. 260-270. 
généralement trois murailles accompagnées de fossés, précédées parfois d'autres lignes vers l'extérieur. Ce dernier type très particulier de fortification peut être dû en partie à la nécessité de tenir à distance les frondeurs ennemis : mais la force supplémentaire donnée par un rempart multiple est une explication suff sante. Il été retrouvé dans la partie sud-ouest de l'Angleterre, qui fait facc à l'Armorique : il est possible que les Vénètes l'y aient introduit dès le II ${ }^{\mathrm{e}}$ siècle av. J.-G. et à nouveau après 56 , s'ils se réfugièrent chez leurs alliés ${ }^{31}$; on n'avan`e plus, toutefois, outre-Manche, cette hypothèse sans quclques réserves : les poteries et les monnaics armoricaines - en général - et Vénètes - en parliculier - sont très rares en Angleterre ${ }^{31}$ bis ; la prise de l'immense Maiden Castle par les réfugiés vénètes est peu vraisemblable; ses enceintes peuvent être plus anciennes; enfin le système de fortification mulliple peut s'être élaboré séparément de chaque côté de la Manche ${ }^{31 t e r}$.

Ces lieux fortifiés se pressent sur la côte armoricaine du Sud et de l'Ouest, Morbihan et Finistère, de l'embouchure de la Loire à la région de Brest ${ }^{32}$. Cela ne signifie point que les Veneti aient occupé tout ce territoire mais qu'ils avaient avec leurs voisins, les Namnetes et les Osismi, un système de défense commun, adapté à un terrain de même nature. Les limites entre ces trois peuples ne sont pas connues avec certitude et il est possible que les Vénètes, avant la catastrophe de 56 , aient cu un territoire beaucoup plus vaste ${ }^{33}$; nous ne préciserons pas l'attribution des forteresses et considérerons comme "vénète ", en hommage à ce peuple qui l'a illustré devant l'Histoire, ce système de défense qu'il a partagé avec ses voisins.

a) Enceintes à remparts multiples:

(28) Beuzec-Cap-Sizun : Castel Coz (Finistère) : sur la côte sud de la baie de Douarnenez, ce camp, le plus anciennement exploré, sert de prototype à la série; inv. B.S.P.F. XI (1914), liste XXXI, $156 ;$ D.A.G., Sizun. Fouilles méthodiques 1869, objets néolithiques et de La Tène III ${ }^{34}$. Trois remparts avec fossés, le principal étant revêtu de pierres; une ligne de défense antérieure (néolithique?); trois lignes supplémentaires à l'extérieur. 90 ares. Fonds de cabane avec objets en majeure partie de La

(31) Cf. R. E. M. Wrifr.ter, Archaeological Journal, CVI, Supplément (1952), p. 75. 200 .

(31 bis) Pour les monnaies, cf. J.-B. Colberl de Beaulicu, Annales de Brelagne, LXI, 1954, p. 184-

(31 ler) V. le compte-rendu, d'ailleurs fort élogieux, de $\Lambda$. L. I. Rivet, Cross Channel, The archaeological Neus Leller, VI, 1958, p. 210-212, avec de précieuses références.

(32) Carte, fig. 1, p. 5, avec indication des forteresses à remparts multiples.

(33) V. la mise au point de P. MERLAT, art. Veneli de la Real-Encyclopüdie, VIII (195), et l'attribution à ce peuple d'un monnayage remarquable par J.-B. Colbert de Beaulieu, "Une énigme de la numismatique armoricaine : les monnaies celtiques des Vénètes ", Mémoires de la soc. d'Hist. el d'arch. de Brelagne, XXXIII (1953).

(34) Plan, pl. XLV, A ; vases, fig. 24, p. 86 (Musée de Guénolé). 
Tène III ; balles de frondes en pierre. Enceintes comparables en Angleterre : Trevelgue, près de Newquay (type "South-Western B »).

(2\%) Cléden-Cap-Sizun : Castel Meur (Finistère) : à 4 kilomètres à l'O.-N.O. de Cléden-Cap-Sizun. à 13 kilomètres à l'Ouest de Castel Coz; inv. B.S.P.F. XI (1914), liste XXXI, $156 ;$ D.A.G., Cléden-Cap-Sizun. Fouilles 1889. Trois remparts avec fossés, peut-être traces d'un quatrième. 2 ha. 95 fonds de cabane de forme rectangulaire ou ovale, poteries, armes, bijoux, balles de fronde (au Musée des Antiquités Nationales).

*(20) Penhars : Kercaradec (Finistère) : à 3 kilomètres à l'Ouest de Quimper, dominant la vallée d'un affluent de l'Odet; inv. B.S.P.F. XI (1914), liste XXXI, 158 ; D.A.G., Penhars. Fouilles $1938^{35}$. Trois remparts avec fossés, le principal construit en pierre sur le roc nivelé, avec palissade vers l'intéricur el gradins d'accès (pour les profodeurs probablement) comme au murus Gallicus de Murcens (Lot) et à l'enceinte des Césarines (près Saint-Céré, Lot). $\mathbf{2}$ ha, 23 a. Poleries remarquables de la première moitié du Ier siècle av. J.-C., et de tradition hallstaltienne avec cordon tressé intérieur, qui se retrouvent en abondance dans le Sud de la Grande-Bretagne ${ }^{36}$; cachette de plusieurs milliers de balles de fronde. Type de camp sud-armoricain, réplique à l'intérieur de terres du camp de falaise maritime. Comparer, en Angleterre, le rempart à gradins de Gurnard's Head (Penwith, Cornwall) ; moins exactement, les remparts composites de pierre (Worlebury, Somerset; autres en France et en Allemagne, cf. Déchelette, Manuel, III, p. 191, fig. 270).

*(16) Ile de Groix : Camp de César (Morbihan) : à 500 mètres au Sud du village de Kervédan, à l'cxtrémité ouest de l'île; inv. B.S.P.F. XIV (1917), liste I.VIII, 456 ; D.A.G., Groix. Sondages $1939^{37}$; complexes de défenses, trois levées, cinq fossés dont certains furent ensuite comblés pour l'établissement d'une grande levée extérieure avec fossé. 81 ares. Poterie gauloise, balles de fronde.

*(4) Sauzon (dans Belle-Ile-en-Mer) : La Pointe du Vieux Château (Morbihan) : à l'extrémité nord de l'île ; inv. B.S.P.F. XIV (1917), liste LVIII, 455. Sondages $1939^{38}$ : trois petits remparts avec fossés. De $\mathbf{4}$ a $\mathbf{5}$ ha, ampleur due à la disposition du promontoire. Poterie gauloise, balles de fronde.

(1) Le Pouliguen : Pen Château (Loire-Atlantique) : sur la côte ouest de la baie de La Baule, aujourd'hui recouvert par un faubourg du Pouliguen ; inv. B.S.P.F. XI (1914), liste XLIV, $290 ; D . A . G$., Pouliguen (Le). Quatre remparts de terre et galets avec fossés. \% ha. : c'est le plus grand de la série.

(6) Saint-Avé : Camp de Villeneuve, Castel Ker Neué (Morbihan) : à Vannes, avec vue sur le golfe; inv. B.S.P.F. XIV (1917), liste LVIII, 459. Trois remparts avec fossés. 20 ares. Fonds de cabanes circulaires, tuiles romaines. Nous intégrons cette enceinte, d'où l'on a une large vue sur le littoral, à la série, bien qu'elle soit plus à l'intérieur des terres que toutes les autres ${ }^{39}$.

(35) Compte rendu des fouilles, p. 54-61, plan et coupe (pl. XXVI-XXVII) ; poteries, fig. 11-12.

(36) Liste et carte des poteries semblables en France et en Grande Bretagne, p. 56-61, fig. 12.

(37) Bref compte-rendu, p. 106. Rapport, v. note précédente. Rapport plus développé : L. MurrayThreipland, Excavations in Brittany, Spring, 1939, Archaeological Journal, C (1943), p. 128-409.

(38) Bref c. r., p. 103.

(39) Exclue pour cette raison de la série maritime, cf. p. $4, n^{\circ} 2$. 
(24) Pont-Croix : Menei Castel (Finistère) : à 2 kilomètres d'Audierne, sur l'estuaire du Goyen ; inv. B.S.P.F. XI (1914), liste XXXI, 15939bis. 'I'rois remparts avec fossés et peut-être un quatrième. Moins de $\mathbf{4 0}$ ares. Objets gallo-romains trouvés en 1889 ?

(18) Clohars-Carnoët : Kergastel (Finistère) : à 5 kilomètres à l'Est de CloharsCarnoët, dans l'embouchure de la Laita ; inv. B.S.P.F. XI (1914), liste XXXI, 156 ; $D . A . G$. , Clohars-Carnoët. Trois remparts, un principal et deux plus légers, avec fossés correspondants. 1 ha, 22.

(30) Pointe de Lostmarc'h (Finistère) : à l'entrée nord de la baie de Douarnenez, sur la côte ouest de la Pointe, à 5 kilomètres au Nord du Cap de la Chèvre ; signalé par P.-R. Giot, Gallia, VII (1949), "Informations", p. 254. Deux remparts avec fossés. Moins de 80 ares. Sépultures néolithiques, peut-être fonds de cabane.

N. B. (33) Ploumoguer (Finistère) : deux promontoires constituent la péninsule de Kermorvan et sont défendus par un complexe peu net de remparts qui paraissent être antiques; inv. B.S.P.F. XI (1914), liste XXXI, 159; D.A.G., Ploumoguer. "Allée couverte \%. - Un fort à remparts multiples est connu à Guernesey, sur la péninsule de Jerbourg ${ }^{40}$.

b) Enceintes à remparl simple:

(3) Saint-Pierre, dit de Quiberon (Morbihan) : à Beg-Naud, à l'angle N.-(). de la péninsule de Quiberon; fouilles de l'abbé Collet 1867 : clous, poteries de La Tène (Musée de Vannes). Le rempart a environ 500 mètres de long. Superficie non indiquée.

(5) Sauzon : île de Mastellic ou de Hastellic, canton de Belle-Ile-en-Mer (Morbihan): à 2.400 mètres de la Pointe du Vieux Château de Belle-Ile (côte ouest). Un peu plus de 40 ares.

(14) Nostang (Morbihan) : à l'Ouest de la route de Nostang à Sainte-Hélène, près de l'estuaire de l'Etel ; inv. B.S.P.F. XIV (1917), liste LVIII, 458. 4 ha.

*(15) Plouhinec (Morbihan) : à la sortie du village de Vieux-Passage, sur le promontoire oriental du port, dominant la rivière d'Etel; inv. B.S.P.F. XIV (1917), liste LVIII, 548. Sondages 1939 à l'intérieur ${ }^{41} .1$ ha, 13. Tessons de la première moitié du Ier siècle av. J.-C., perle en verre bleu, balles de fronde.

(19) Ergué-Armel : Beg-ar-Castel (Finistère) : à 300 mètres au Sud du Château de Lanroz, dans l'estuaire de l'Odet; inv. B.S.P.F. XI (1914), liste XXXI, 157. 80 ares. Traces d'incendie et peut-être de vitrification.

(21) Gouesnach (Finistère) : à Saint-Gadou, à 4 kilomètres au N. de Gouesnach, exactement en face du précédent sur l'estuaire de l'Odet; inv. B.S.P.F. XI (1914), liste XXXI, 157. De 1 ha, 25 à 1 ha, 50.

(39 bis) La référence indiquée à $B . S . P . F$. XI, 1914, liste XXXI, p. 159 paraît plutôt concerner (par référence à B.S. P.F. III, 1906, p. 366) le camp de Castellien (commune de Meilars), non mentionné ici. Il s'agit probablement ici d'un camp de promontoire situé à 150 mètres au S.-O. d'un étarlissement romain occupant les terres de Kervénénec, d'après la référence aux Epoques préhistoriques... du Finislère de P. du Chatellier (1907), p. 304, comme me le suggère M. P. Merlat, Directeur de la circonscription de Rennes, qui a bien voulu relire cet article sur épreuves ainsi que M. Ernest Will, Directeur de la circonscription de Lille, et le $\mathrm{D}^{\mathrm{r}}$ Colbert de Beaulieu. Qu'ils trouvent ici mes vifs remerciements.

(40) Cf. p. 102, no 1 ; Jacquetta Hawkes, The Archaeology of the Channel Islands, II (1937), p. 192193 ; T. D. KENDrick, The Archaeology of the Channel Islands, I (1928), p. 177-189.

(4I) Bref compte rendu, p. 106. Rapport, v. plus haut, n. 37. 
(25) Primelin (Finistère) : à l'Ouest du village du Castel ; inv. B.S.P.F. XI (1914), liste XXXI, $159 ;$ D.A.G., Primelin. 80 ares. Peul-êlre oulillage de silex.

(26) Plogoff (Finistère) : à l'extrémité de la Pointe du Raz; inv. B. S.P.F. XI (1914), liste XXXI, 158;D.A.G., Plogoff. 2 ha, 43. Découvertes fréquentes de pointes de flèche barbelées en silex.

(29) Beuzec-Cap-Sizun (Finistère) : à l'Ouest de Castel-Coz (v. plus haut, no 28); inv. B.S.P.F. XI (1914), liste XXXI, 156. Moins de 20 ares.

N. B. - (2) Arzon (Morbihan) : promontoire de la baie du Morbihan, en face du Moulin de Pencastel ; inv. B.S.P.F. XIV (1917), liste LVIII, 455. Rempart de sable à revêtement extérieur de pierre. 1 ha, 21. Antique ou médiéval? - (33) Le petit promontoire (v. plus haut, N. B., no 33 ) est peut-être à rempart simple. - A Jersey a été reconnu un fort de hauteur à rempart simple, un autre existe peut-être ${ }^{\mathbf{2}}$.

Ces forts "vénètes", au nombre d'une vingtaine, sont tous situés sur le littoral ou à proximité immédiatc, dans les estuaires. Ils sont pour la plupart exigus. Les plus puissamment défendus, par des lignes multiples, ont leur pendant dans la Grande-Bretagne méridionale, bien qu'ils s'élèvent sur la côte sud de l'Armorique, non sur la côte nord qui fait face à la grande île : c'est qu'ils appartenaient à la vaste confédération maritime dont les Veneti, établis sur cette côte méridionale, étaient les maîtres. Les plus puissantes de ces enccintes n'auraient-elles pas été construites contre l'attaque de Brutus, en 56 ?

\section{Forteresses secondaires, non maritimes pour la plupart}

Le reste des enceintes, de faible superficie, est en grande majorité à l'intérieur des terres et réparti chez différents peuples, en plus grand nombre toutefois à l'Ouest de la Seine (les $5 / 6^{\mathrm{e}}$ environ) ${ }^{43}$. Elles sont du type traditionnel à rempart et fossé simples, sans murus Gallicus et sans le fossé plat ni la grande entrée des enceintes « belges ». Elles s'élèvent sur des hautcurs pour la plupart.

Veneli :

(7) Plumelec : Camp du Château blanc (Morbihan) : à 2 kilomètres au S.-S.0. de Plumelec, dominant la Claie; inv. B.S.P.F. XIV (1917), liste LVIII, 459 ; D.A.G., Plumelec. 2 ha.

(9) Saint-Aignan : Castel Finans (Morbihan) : à 2 kilomètres au S.-S.O. de Murde-Bretagne, sur le cours supérieur du Blavet; inv. B.S.P.F. XIV (1917), liste LVIII, 459. Deux remparts avec fossé intermédiaire : le rempart intérieur est, sans doute, soit la muraille et la limite primitives, soit l'effet d'une réduction du camp. 4 ha.

(11) Bieuzy (Morbihan) : au Sud de Castennec, dans une boucle du Blavet, en aval du précédent, à 50 mètres au Nord de la Chapelle de la Sainte Trinité ; inv. B.S.P.F.

(42) Cf. p. 102, n. 1 et J. Hawkes, o. c.

(42) Carte, pl. XLIII, p. 103. 
XIV (1917), liste LVIII, 455. De 6 à 8 ha. La superficie, forte pour la série, est due à la forme du promontoire.

(13) Quistinic (Morbihan) : dominant le Blavet en aval du précédent, au confluent d'un cours d'eau tributaire ; inv. B.S.P.F. XIV (1917), liste LVIII, 459. "Grand " mais en majeure partie abrupt et peu habitable.

(10) Langonnet (Morbihan) : à 2 kilomètres au Sud de l'abbaye, surplombant un bras de l'Ellé ; inv. B.S.P.F. XIV (1917), liste LVIII, 456.80 ares.

(17) Arzano : Camp de César (Finistère) : près de Saint-Adrien, à 4 kilomètres à l'Est de Quimperlé, dominant le cours supérieur de l'Ellé ; inv. B.S.P.F. XI (1914), liste XXI, 156. Rempart intérieur, d'une époque différente, à l'exlrémité du promontoire. De 80 ares à 1 ha, 25.

(22) Plonéour-Lanvern (Finistère) : à 400 mètres au S.-O. de la ferme de Kervelan; inv. B.S.P.F. XI (1914), liste XXXI, 158; D.A.G., Plonéour-Lanvern. Au moins $1 \mathrm{ha}, 60$. Série de tumulus.

(23) Plonéour-Lanvern (Finistère) : à l'Est de la ferme de Kergoulouarn; inv. B.S.P.F. XI (1914), liste XXXI, 159; D.A.G., Plonéour-Lanvern. Poterie "gauloise ". Comparable au camp de Hottot, dans le Calvados (plus loin, no 52), ce camp, presque rectangulaire avec trois côtés rectilignes, peut être «l'œuvre d'un Gaulois qui avait vu des campements romains ». Superficie non indiquée.

N. B. - (12) Plouay (Morbihan) : à l'Ouest du village, dominant la Scorff, deux remparts dont l'un, le plus extérieur, contient probablement un rempart antique avec fossé et contrescarpe ; inv. B.S.P.F. XIV (1917), liste LVIII, 458. 28 ha. : cette superficie, près de quatre fois plus forte que celle des plus grands forts de la série, pourrait être due, comme à Sauzon (Belle-Ile-en-Mer ; plus haut, no 4), à la conformation du terrain, mais l'antiquité de l'enceinte reste à prouver et la possibilité doit être réservée de l'inclure dans le groupe des grands oppidums.

Osismi:

(32) Sizun : Castel Doun, Castel Longue (Finistère) : à 2 kilomètres au N.-O. de l'église, sur le cours supérieur de l'Elorn; inv. B.S.P.F. XI (1914), liste XXXI, 160 D.A.G., Sizun. De 2 ha, 85 à 3 ha, 25.

(34) Dirinon : Castellic (Finistère) : à 2 kilomètres au N.-O. du village, sur l'Elorn, au début de l'estuaire ; inv. B.S.P.F. XI (1914), 157 ("Goaren-ar-G'hastel ») ; D.A.G., Dirinon. Moins de 20 ares.

(35) Le Folgoüt : Castel Pen-Lédan, Castel Bras-Landivern, Camp de César (Finistère) : à $1 \mathrm{~km} .200$ au Sud du Folgoül ; inv. B.S.P.F. XI (1914), liste XXXI, 158. Rempart à revêtement de pierre sèche, traces possibles d'un autre rempart extérieur représentant une extension de l'enceinte. 1 ha, 62. C'est l'enceinte la plus puissante du Finistère occidental.

Coriosolitae:

(36) Plédran : Camp de Péran, Camp romain (Gòtes-du-Nord) : camp circulaire de plateau ; inv. B.S.P.F. VIII (1911), liste provisoire, 131 ; D.A.G., Plédran. Deux remparts avec fossés, le principal à l'intérieur étant vitrifié avec traces de poutrages (mais il ne s'agit pas d'un murus Gallicus). De 80 ares à 1 ha, 25. Cette enceinte n'occupe 
pas une falaise maritime ni une falaise fluviale : malgré sa double ligne de défense, elle n'est pas un exemple, sur la côte nord de l'Armorique, d'un châtcau-fort du typc «vćnc̀tc".

(37) Trémargat (Gôtes-du-Nord) : sur le cours supérieur du Blavet, au confluent d'un petit tributaire, au lieudit Toul Goulic. Près de 5 ha. Beau camp de hauteur, le plus grand des Côtes-du-Nord.

(38) Comblessac : Camp du Mur, Camp des Romains (Ille-et-Vilaine, à la limite du Morbihan) : sur les terres du Château de Marsac, dominant la vallée de l'Aff ; inv. B.S.P.F. XI (1914), liste XXXVII, 278 ; D.A.G., Comblessac. Rempart avec peut-être revêtement de pierre à l'extérieur et traces possibles de deux remparts extérieurs près de l'entrée. $\mathbf{5}$ ha, $\mathbf{5 0}$ : l'une des plus grandes enceintes de la région dans cette série. Temple romain (?) octogonal, monnaies d'Auguste et Tibère, poteries, tuiles.

(39) Les Brulais (Ille-et-Vilaine) : non loin de la vallée de l'Aff, à 1 kilomètre à l'Est du village ; inv. B.S.P.F. XI (1914) liste XXXVII, 279. 3 ha, 65.

Redones:

N. B. - (41) Saint-Brolabre : Le Châtel, La Cour-Baudoin (Ille-et-Vilaine) : dans la partie Ouest de la communne, dominant la plaine côtière. Rempart avec fossé creusé dans le roc. Moins de $\mathbf{2 0}$ ares. L'antiquité de l'enceinte est à vérifier.

\section{Unelli :}

(44) Carolles (Manche) : sur la falaise de mer ; inv. B.S.P.F. XIII (1916), liste LII, $162 ;$ D.A.G., Carolles. Camp de forme carrée irrégulière avec une annexe trianguaire au Nord, peut-être antérieure. " 7 ha. Au Nord, ruines des "Chapelles", peut-être galloromaines.

(46) Jobourg : le Vaux du Câtel, Camp romain (Manche) : sur le Nez de Jobourg; inv. B.S.P.F. XIII (1916), liste LII, 163. Kempart à revêtement extérieur de pierre. 8 ha.

(47) Le Vast : Camp des Castiaux, Camp de Pepinvast, Camp romain (Manche) : sur les terres du château de Pepinvast, près du Vicel, dominant la vallée de la Saire; inv. B.S.P.F. XIII (1916), liste LII, 163. De 2 ha, 85 à 3 ha, 45.

(48) Montebourg : le Petit Moncastre (Mont-Castre) : à 1250 mètres au N.-O. de Montebourg; inv. B.S.P.F. XIII (1916), liste LII, 163. De 1 ha, 20 à 1 ha, 60.

N. B. - Un autre camp est à rechercher à l'Ouest de Barneville (Manche), au Nord du Nez de Carteret, dominant les baies de Carteret et de Port-Bail ; cf. inv. B.S.P.F. XIII (1916), lisge LII, 162 et Gerville, Mém. Soc. Antiquaire de France, VII (1826), p. $183, \mathrm{pl} . \mathrm{V}, 3^{44}$.

\section{Baiocasses :}

(51) Commes : Le Chevalier d'Escures, la Butte (Calvados) : dominant la vallée de l'Aure, non loin du littoral ; inv. B.S.P.F. X (1913), liste XVI, 282 ; D.A.G., Carteret. 3 ha, 25.

(52) Hottot : Camp des Anglais (Calvados) : dominant la Seulles; inv. B.S.P.F. X (1913), liste XVI, 282; D.A.G., Hottot-les-Bagues. Camp oblong comparable à celui de Plonéour-Lanvern (plus haut, no 23). 3 ha, 25.

(44) Cf. p. 116, Nole. 
Viducasses:

(53) Soûmont-Saint-Quenlin : Le Monl-Joly (Caalvados) : contient le tombeau de Marie Joly (peut-être sur un monument mégalithique) et une nécropole mérovingienne ; inv. B.S.P.F., X (1913), liste XVI, 283; D.A.G., Soûmont-Saint-Quentin. 3 ha, 25.

(54) Moult : Camp de la Hogue, Camp d'Ouézy (Calvados) : au N.-E. de Moult; inv. B.S.P.F. X (1913), liste XVI, 283. 4 ha.

\section{Aulerci Eburovices:}

(57) La Courbe : Château Gontier, Les Vieux Châteaux, Les Pierres Brûlées (Orne) : domine un défilé de l'Orne ; inv. B.S.P.F. XIV (1917), liste LXII, 473. Rempart double, avec fossés et contrescarpe extérieure, les murs étant en grands blocs de pierre, en partie vitrifiés (cf. no 58). 2 ha. 85.

N. B. - (58) Montmerrei (Orne) : au château de Blanchelande; inv. B.S.P.F. XIV (1917), liste LXII, $475 ;$ D.A.G., Montmerrei. Rempart en fer à cheval, en grands blocs de pierre, sans fossé visible. 4 ha, 85. L'antiquité de cet ouvrage n'est pas prouvée, ce qui jette peut-être un doute sur celle du no 57 , étant donné sa similitude avec celui-ci.

(59) Igé (Orne) : à Crochemelier ; inv. B.S.P.F., XIV (1917), liste LXII, 474 ; D.A.G., Igé. Fouilles 1875 : clou, épée et lance de bronze, sépultures dans le roc à l'extérieur, tessons d'époque hallstattienne tardive. Superficie très petite ("tiny ») : 50 ares d'après le D.A.G.

(64) Sorel-Moussel : Fort-Harrouard (Eurc-et-Loir) : surveille la vallée de l'Eure ; inv. B.S.P.F. XI (1914), liste XXX, $155 ; D . A . G$., Sorel-Moussel. Fouilles méthodiques de l'abbé Philippe. Rempart de La Tène III. 8 ha. Occupations néolithiques, de la fin du Bronze avec un rempart de craie et de La Tène III avec une enceinte à caractère belge, huttes incendiées (peut-être pendant la Guerre des Gaules?).

Veliocasses:

(60) Bouquelon : Les Forts, Le Mont Finet (Eure) : dominant la vallée de la Risle ; inv. B.S.P.F. XI (1914), liste XXIX, 152. 2 ha.

(66) Saint-Nicolas-de-la-Taille : Le Câtelier, Camp de Boudeville (Seine-Maritime) : à 2 kilomètres au S.-S. O. de l'église de Saint-Nicolas; inv. B.S.P.F. XVI (1919), liste LXXVII, $186 ; D . A . G$., Saint-Nicolas-de-la-Taille. De 1 ha, 20 à 1 ha, 60. Région où s'élèvera Juliobona, chef-lieu et port des Caleti (Lillebonne), aujourd'hui à 4 kilomètres dans les terres par suite de l'ensablement de l'estuaire.

(69) Orival : Le Gâtelier (Seine-Maritime) : colline à côté de la vallée de la Seine, vers le village; Deglatigny, Documents el noles archéologiques, 2 (1927), p. 14-18, plan, pl. III. Visité 1939 et $1954^{45}$. Défenses complexes : deux remparts, l'un principal avec fossé et contrescarpe, l'autre extérieur, plus bas, ce qui est exceptionnel, et remarquable. Superficie non indiquée. Tessons trouvés dans le rempart : fragment à cordon, vases à pied ("sub-pedestal )) du type belge de La Tène III ; lc camp a dû être construit ou reconstruit peu avant la guerre des Gaules. Temple de tradition celtique à l'intérieur (Deglatigny).

(45) V. la notice, p. 123. 
Lexovii:

(55) Cambremer : Château des Anglais (Calvados) : sur le Mont Argis, dominant la Vallée d'Auge; 2 ha, 45. Caumont, Stalislique monumentale du Calvados, IV, p. 158.

Ambiani :

(76) Mareuil-Caubert : Camp de César (Somme) : au S.-o. d'Abbeville, sur le Mont-Caubert, dominant la basse Somme; inv. B.S.P.F., XVII (1920), liste LXIX, $56 ;$ D.A.G., Mont-Caubert. Surveille le passage le plus bas de la vallée, comme Abbeville qui lui a succédé. Si la superficie, " certainement grande ", était préciscée par des sondages au Nord, cette enceinte pourrait être adjointe ì la série des grands oppidums de tỵe traditionnel (plus haut, I, ל).

(80) Chipilly (Somme) : sur la rive gauche de la haute Somme, à 24 kilometres à l'Est d'Amiens, au coude que fait le fleuve vers le sud; inv. B.S.P.F. XVII (1920), liste I.XXIX, 56. 10 ha.

Bellocaci:

(82) Catenoy (Oise) : à $10 \mathrm{~km} .500$ à l'E.-S.L. de Clermont, entre Catenoy et Sacy-le-Grand ; inv. B.S.P.F. XIV (1917), liste LXI, 469; D.A.G., Catenoy. Fouilles nombreuses. Malgré la forme arrondie du fossé et la présence de pierres en grande quantité dans le mur, n'est pas du type "belge ". De 4 ha, 50 à 5 ha.

Suessiones:

(90) Pont-Saint-Mard (Aisne) : sur la Montagne de Plain-Chatel, à l'angle de la voie romaine Soissons-Saint-Quentin et de l'Ailette; Marville, Bull. Soc. Antiquaires de Picardie, VIII (1862-1864), p. 108, plan sommaire. 8 ha.

Ce qui frappe tout d'abord à l'issue de cet inventaire, c'est la densité des ouvrages de défense, bien mise en lumière par la carte générale, et leur répartition qui correspond aux théâtres d'opération de la guerre des Gaules, notammenl du début : en Armorique et en Belgique, y compris le sursaut de う̌l dans cette dernière région. Nous voyons maintenant, inscrite sur le terrain, la défense littorale des Vénètes vivant sur mer, et, en pays belge, la formidable et soudaine surrection de forteresses d'un type nouveau. Il faut lire, sur ce sujet, les belles pages de la première partic de l'ouvrage, qui évoquent, d'une part, la puissance toute maritime des Vénètes accrochés à leurs repaircs "suspendus dans des brumes persistantes, entre les cris mornes des oiseaux de mer et le fracas sans répit des brisants ", "refuges exigus d'un peuple dont le mode de vie était plutôt éparpillé sur la mer que centré sur un foyer terrestre ", - d'autre part, le fort potentiel de guerre d'un Galba, roi des Suessions, chef de cinquante mille hommes en armes, maître de douze oppidums dont le plus puissant, Noviodunum, a défié les machines de César. Les étroites relations des Vénètes avec les Brittons du 
Cornwall sont évoquées de façon non moins vivante par les fortifications-sœurs construites, peut-être par leurs agents et leurs réfugiés, chez leurs alliés, sur cette còte sud-occidentale de la grande île.

Certes, l'origine continentale des défenses multiples de Grande-Bretagne ne découle pas avec certitude de cette enquête. Ni Huelgoat, ni Le Petit-Celland, ne sont l'équivalent de Maiden Castle et unc enquête poussée en Angleterre même, par excmple dans le Wessex, révélerait peut-être des prototypes aussi valables que les minuscules places fortes de la còte méridionale du Morbihan. C'est surtout sur les forteresses gauloises, grandes et petites, que cet ouvrage nous apporte du nouveau. Par exemple, la distribution des grands oppidums est particulièrement suggestive. Certains apparaissent, à l'évidence, comme des chefs-lieux de peuplade, établis antérieurement à l'époque de la menace romaine : Castillon des Baiocasses, Saint-Désir des Lexovii, le Mont César des Bellovaci, Pommiers (Noviodunum) des Suessiones, Etrun des Alrebates, Vermand des Viromandui, Vieux-Reims des Remi, Vieux-Châlons des Catuvellauni. D'autres se présentent comme des points de ralliement créés par la menace romaine ou, tout au moins, comme fortifiés à cette époque : Le Petit-Celland, Guégon, IIuelgoat, Fécamp et les oppidums de type belge, certains n'ayant fait l'objet que d'une occupation éphémère. On ne saurait toutefois ériger la règle d'un grand oppidum unique par peuplade et, sur ce point, la carte de ces enceintes majeures (pl. I de l'ouvrage) paraît un peu limitative : il peut y avoir eu deux ou mème plusicurs oppidums importants chez un même peuple, notamment lorsque son territoire est étendu et comporte un front de mer. Le classement qu'on vient de parcourir rectifiant en quelques points celui, trop strict à mon sens, des cartes qui en expriment néanmoins l'essentiel, corrige cette impression en comptant notamment au nombre des places-fortes essentielles toutes celles auxquelles on reconnait la technique du murus Gallicus.

Il ne serait pas moins instructif de récapituler le nombre et les divers genres de forteresses dont chaque peuple a disposé à l'époque de la Guerre des Gaules. L'imprécision trop fréquente des limites interdit d'en faire un décompte exact mais le classement précédent suggère, d'une catégorie à l'autre, quelques groupements impressionnants sur un mème territoire : tel celui des Véliocasses avec ses six enceintes de type belge, dont l'immense Saint-Samson-de-la-Roque ( $n^{0} 61$ ), son grand oppidum du Mont Calidu $\left(n^{0} 68\right)$, ses quatre ou cinq autres camps secondaires. Certes, la valléc de la Scine, à la jonction des deux Gaules, élait unc artère particulièrement puissante. Nais il est remarquable que d'autres vallées moins importantes aient eu aussi leur chapelet de forteresses, que ce soient les rivières armoricaines, la Somme, ou l'Aisne. D'une façon générale, toutes 
ces enceintes, quand elles ne sont pas purement maritimes, surplombent une vallée, un vallon ou un esluaire et lorsque, par exception, elles s'élèvent en plaine, c'est dans la boucle d'une rivière : forteresses de hauteur, presque toutes ; mais aussi, guette ou verrou, d'une voie fluviale, le plus souvent.

Un certain nombre de ces oppidums, surgis ou fortifiés pour la plupart en 57-56, ont survécu à la tourmente; d'autres n'étaient que fortifications de circonstances. On peut notamment rapporter au sursaut de 51 les fortifications réduites de Huelgoat $\left(\mathrm{n}^{0} 31\right)$ et de Guégon ( $\left.\mathrm{n}^{\circ} 8\right)$, quartiers-généraux respectifs des Osismes et des Curiosolites. En plusieurs lieux marqués par la défense, la vie a continué : "pendant une génération, sous la surveillance que Rome a jugée nécessaire ou réalisable, nous pouvons imaginer les membres de ces peuples encore cantonnés dans leurs grossiers baraquements et même sur leurs oppida de sommet (cf. B.G. II, 28 à propos des Nerviens), frappant toujours leurs propres monnaics " (p. 21). Ainsi ont survécu en Gaule, Alésia mise à part : Fécamp, La Cité de Limes, Pommicrs, Saint-Marcel-de-Félines (Le Crêt-Chatelard), Essalois, l'Impernal, Bibracte, Villejoubert, le Camp de la Ségourie, Jœuvres, jusque vers le début de notre ère ; en Bretagne, Maiden Castle (Dorsetshire) a survécu trente ou quarante années à la catastrophe de 44 après J.-C.; en plein I ${ }^{\mathrm{er}}$ siècle, la technique des remparts "belges" de l'époque de la guerre des Gaules renait en Bretagne belge, à la veille de l'invasion romaine de 43 , à Oldbury dans le Kent ; la technique du murus Gallicus renait semblablement en Écosse, à Burghead, probablement à la fin du $\mathrm{I}^{\mathrm{er}}$ siècle, devant l'invasion dirigée par Agricola ${ }^{46}$.

Grâce à cet enquête, l'inventaire des enceintes gauloises est fait pour une partic de la Gaule et deux types nouveaux de fortification, l'un à lignes multiples, l'autre à large fossé, sont reconnus ainsi que l'importance de la fronde dans les combats de ce temps. La chronologie de ces ouvrages est en partie fixée, au moins pour la période de la guerre des Gaules, et pour celles qui la précèdent ou la suivent d'assez près. L'occupation souvent très ancienne, parfois néolithique, de plus d'un site, est désormais prouvée. Il reste à préciser l'ancienneté des plus vastes de ces forteresses, qui ont toutes chances d'avoir été les chefslieux d'importantes peuplades : c'est la question de la mise en place des cités gauloises au cours du II e Age du Fer qui se trouve posée sur des bases nouvelles. Je ne serais pas étonné que des recherches, qui restent à faire systématiquement, sur les monnaies et les tessons de sites désormais précisés par cet important travail et dont plus d'un pourraient être explorés avec fruit, permettent de distinguer :

10 les foyers centraux occupés le plus anciennement, fortifiés ou non ;

(46) Cf. p. xv, 12, 77; 176 . 
$2^{0}$ les fortifications de ces foyers ou de siles secondaires provoquées directement ou rétrospectivement par l'invasion des Cimbres et des Teutons, qui, un demi-siècle avant l'invasion romaine, a dù susciter la surrection d'une bonne partie de ces enceintes, peut-être un certain nombre de celles que caractérise un murus Gallicus ;

$3^{\circ}$ les défenses nées de la Guerre des Gaules;

$4^{0}$ des remparts d'époque gallo-romaine précoce, comportant même lo murus Gallicus.

Paul-IIarie Duval. 


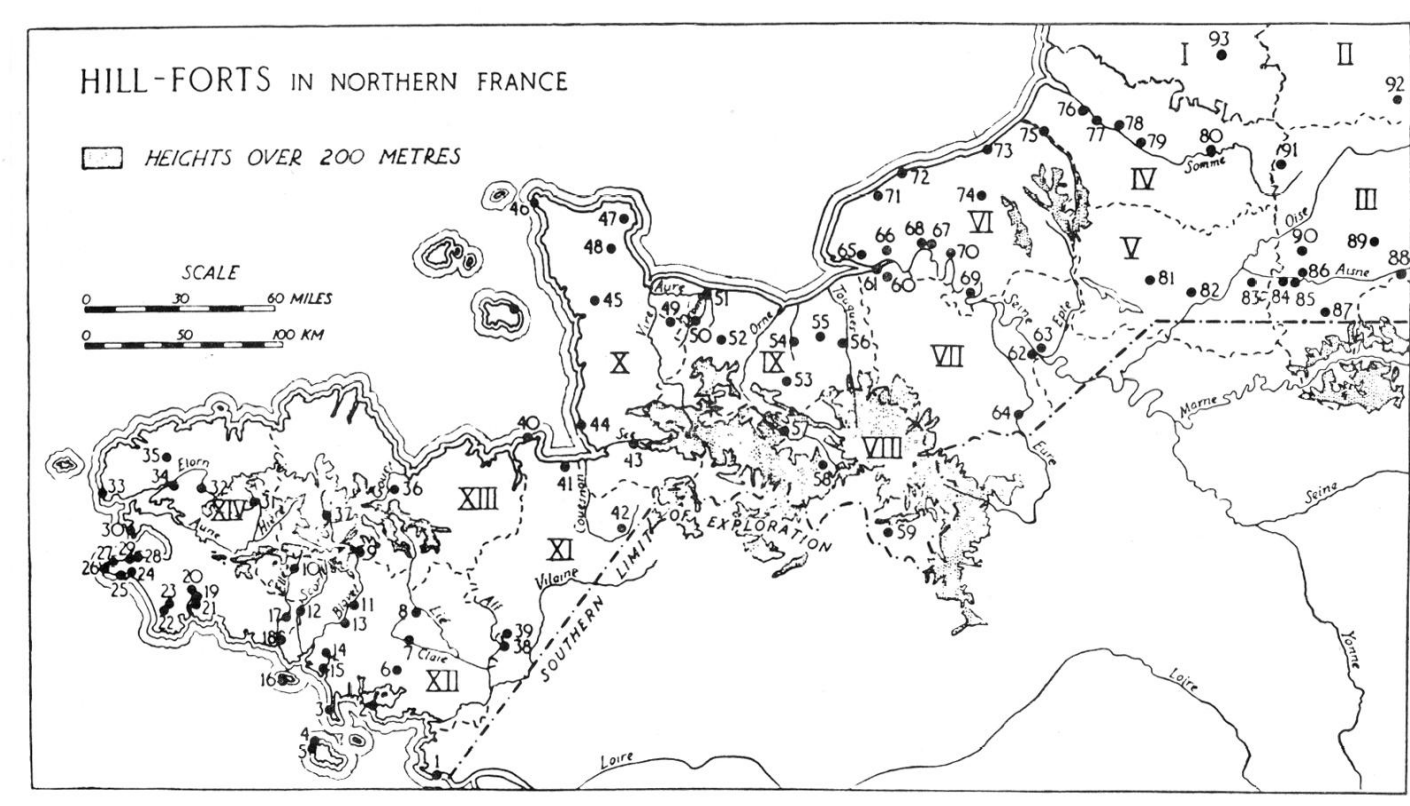

Fig. 2. - Carte des enceintes gauloises inventoriées ou touillées dans les départements suivants :

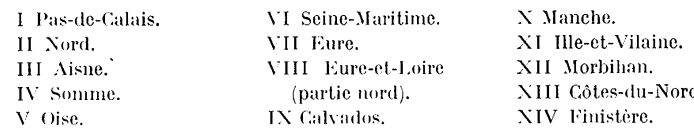

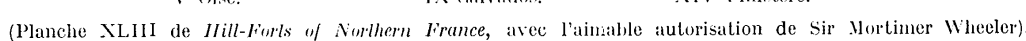

